\title{
Investor's Optimal Strategy with and Without Transaction Cost Under Ornstein-Uhlenbeck and Constant Elasticity of Variance (CEV) Models Via Exponential Utility Maximization
}

\author{
Silas Abahia Ihedioha ${ }^{1, *}$, Nanle Tanko Danat ${ }^{1}$, Audu Buba ${ }^{2}$ \\ ${ }^{1}$ Department of Mathematics, Plateau State University Bokkos, Jos, Plateau State, Nigeria \\ ${ }^{2}$ Department of Actuarial Science, University of Jos, Jos, Plateau State, Nigeria \\ Email address: \\ silasihedioha@yahoo.com (S. A. Ihedioha), danatnanle@gmail.com (N. T. Danat), bubakaudu@gmail.com (A. Buba) \\ ${ }^{*}$ Corresponding author
}

To cite this article:

Silas Abahia Ihedioha, Nanle Tanko Danat, Audu Buba. Investor's Optimal Strategy with and Without Transaction Cost Under Ornstein-Uhlenbeck and Constant Elasticity of Variance (CEV) Models Via Exponential Utility Maximization. Pure and Applied Mathematics Journal. Vol. x, No. x, 2020, pp. x-x. doi: 10.11648/j.pamj.20200903.12

Received: June 5, 2020; Accepted: June 20, 2020; Published: July 4, 2020

\begin{abstract}
In this work, we studied the optimal investment problem of an investor who had exponential utility preference and traded two assets; (1) a risky asset which price dynamics was governed by the Constant Elasticity of variance (CEV) model and (2) a risk-free asset which price system followed the Ornstein-Uhlenbeck model. We employed the maximum principle of dynamic programming to obtain the Hamilton-Jacobi-Bellman (H-J-B) equation on which the first principle and the elimination of variable dependency were applied to get the closed-form of the investor's optimal strategies. Two scenarios where the Brownian motions correlated and where they did not correlate were investigated. Also considered were the cases of when transaction cost was involved and when transaction cost was not involved. This lead to six cases that among the results obtained was that the investor has an optimal investment strategy that requires more amount of money for investment when the Brownian motions do not correlate and there is transaction cost than when the Brownian motions correlate and there is no transaction.
\end{abstract}

Keywords: Investor, Optimal Strategy, Transaction Cost, Ornstein-Uhlenbeck Model, Constant of Elasticity of Variance (CEV) Model, Exponential Utility Maximization

\section{Introduction}

The problem of utility maximization is of great importance. This has lead to many researchers in financial mathematics to greatly focus on solving optimal investment problem of utility maximization.

This paper intends to find the optimal investment strategy for an investor who participates in a financial market, in which the interest rate of the risk-free asset is stochastic and governed by the Ornstein-Uhlenbeck model and the risky asset assumed to follow the constant elasticity of variance (CEV) model and look into the variation that would occur when the Brownian motions correlate and when the Brownian motions do not correlate as well as find what happens when transaction cost is charged.

To achieve this goal we reviewed some works scholars have done, especially recent ones. Merton is among the early researchers in this area and one of his works is [1] where he used the stochastic optimal control method in continuous finance to obtain a closed form solution to the problem of optimal portfolio under the specific assumptions about the assets returns and the investor preferences these days insurance company invest both money market and stocks market. Due to high rate involved in the stock market investment strategies and risk management are becoming more important.

$\mathrm{Gu}$ et al. [2] worked to obtain optimal strategies and optimal value functions under constant elasticity of variance (CEV) model on the condition that the insurer purchased excess-of-loss reinsurance.

The optimal reinsurance investment problem for an insurer with jump diffusion risk process under the constant elasticity of variance (CEV) model was worked on by [3].

Zhao and Rong [4] extended the efforts made by looking into portfolio selection problem with multiple risky assets under the constant elasticity of variance (CEV) model. 
The work [2] is on the optimization of defined contribution (DC) pension fund scheme in which they obtained a closed-form solution for the optimal investment strategy under a constant elasticity of variance model and power and exponential utility preferences

Liu et al. [3] worked on optimal investment problem under the constant elasticity of variance (CEV) model for utility maximization where taxes, dividends and transaction are involved.

In the work of [2] optimal investment problem was studied and they obtained optimal strategies and value functions under a constant elasticity of variance model based on the fact that the insurer can purchase excess-of-loss reinsurance.

Asset and liability management problem with stochastic interest rate in which the interest was assumed to be an affine interest rate model was the study that [5] carried out.

Ihedioha et al. [6] studied the effect of correlation of Brownian motions on an investor's optimal investment and consumption decision under Ornstein-Uhlenbeck Model. They applied the maximum principle to obtain the HJB equation for the value function. The HJB equation derived was transformed into an ordinary differential equation; specifically, the Bernoulli equation, using elimination of dependency on variables and tackled the problem.

Further reviewed works were those on pensions that were related to the ones already given.

Okonkwo et al. [7] examined the optimal investment strategy for a defined contribution (DC) pension scheme that was modeled such that the fund was invested partly in riskless assets and partly in risky assets and that the market has a constant interest rate, a stochastic volatility that follows the Heston model. The salary was assumed to be constant over the entire career of the Pension Plan Participant (PPP) and the contribution was a constant proportion of the salary. They used the CRRA utility function to obtain the Hamilton-Jacobi-Bellman (HJB) equation which was solved by the Prandtl Asymptotic Matching Method to get the required investment strategy.

Osu et al. [8] in their study developed a pension fund management strategies in a DC scheme for the distribution phase. In this, the Pension plan member (PPM) was allowed to invest in a risk-free and a risky asset, under the constant elasticity of variance (CEV) model. The constrained optimization program that was developed was transformed into a nonlinear partial differential equation, using the associated Hamilton Jacobi Bellman equation from which the explicit solution of the constant relative risk aversion (CRRA) is obtained, using Legendre transform, dual theory, and change of variable methods. They established this with a proposition that the elastic parameter, $\beta$, say, must not necessarily be equal to one $(\beta \neq 1)$. They also constructed and proved a theorem on the pension investor's wealth investment strategy. They used sensitivity analysis to unveiled the dangers of CRRA utility options during the period after retirement

Akpanibah et al. [9] studied the optimal investment strategies for a plan contributor in a defined pension scheme, with stochastic salary and extra contributions, under the affine interest rate model. They considered two cases where the extra contribution rates were stochastic and constant respectively. Three different assets namely risk free asset (cash), zero coupon bonds and the risky asset (stock) were considered. They obtained the optimal investment strategies for the three investments using Legendre transformation method and dual theory where exponential utility function for two of the cases was involved and found result that showed that the strategies for the respective investments when there was no extra contribution could be used when the extra contribution rate was constant but could not be used when it was stochastic. This clearly gave the member and the fund manager good insight on how to invest to obtain a maximum profit with minimal risk.

Njoku et al. [10] studied the effect of extra contribution on stochastic optimal investment strategies in a DC pension with stochastic salary under affine interest rate model.

Wang and Chen [11] investigated a defined contribution (DC) pension plan investment problem during the accumulation phase under the multi-period mean-variance criterion.

Osu et al. [12] studied optimization problem with return of premium in a DC pension with multiple contributors.

Osu et al. [13] studied optimal investment strategies in DC pension fund with multiple contributions using Legendre transformation method to obtain the explicit solution for CRRA and CARA.

Osu et al. [14] investigated the effect of Inflation and the impact of hedging on the optimal investment strategies for a prospective investor in a DC pension scheme, using inflation indexed bond and inflation-linked stock. Their model permitted the plan member to make a defined contribution, as provided in the Nigerian Pension Reform Act of 2004. The pension plan member was allowed to invest in risk-free asset (cash), and two risky assets (i.e., the inflation-indexed bond and inflation-linked stock). A stochastic differential equation of the pension wealth that took into account certain agreed proportions of the plan member's salary, paid as contribution towards the pension fund was constructed. The Hamilton-Jacobi-Bellman (H-J-B) equation, Legendre transformation, and dual theory were used to obtain the explicit solution of the optimal investment strategies for CRRA utility function.

Njoku and Osu [15] worked on the optimal pension wealth investment strategy during the decumulation phase, in a defined contribution (DC) pension scheme where the pension plan member was allowed to invest in a risk free and a risky asset, under the constant elasticity of variance (CEV) model. The explicit solution of the constant relative risk aversion (CRRA) and constant absolute risk aversion (CARA) utility functions were obtained, using Legendre transform, dual theory, and change of variable methods. They established that the elastic parameter, $\beta$, say, must not necessarily be equal to one $(\beta \neq 1)$.

It can be easily seen that most of the works reviewed did not discuss transaction cost hence our choice of this topic.

\section{Methodology}

\subsection{Brownian Motion}

Brownian motion is regard as a simple continuous 
stochastic process that is widely used in finance and physics for modelling random behaviour that evolves over time. An example of such behaviour is the random movements of molecules of gas or fluctuation in an assets price.

In mathematics, Brownian motion is described by Wiener process as a continuous-time stochastic process named in honour ofNorbert Wiener.

The Wiener process $W_{t}$ is characterized by four facts, and these are;

1. $W_{0}=0$

2. $W_{t}$ is almost surely continous

3. $W_{t}$ has independent increments

4. $W_{t}-W_{s} \sim N(0, t-s),($ for $0 \leq s \leq t)$

Note: $N\left(\mu, \sigma^{2}\right)$ denotes the normal distribution with expected value of $\mu$ and variance $\left(\sigma^{2}\right)$. The condition that it has independent increment means that if $0 \leq s_{1} \leq t_{1} \leq s_{2} \leq$ $t_{2}$ then $W_{t 1}-W_{s 1}$ and $W_{t 2}-W_{s 2}$ are independent random variables.

\subsection{Ornstein-Uhlenbeck Model}

The Ornstein-Uhlenbeck process is one of the several approaches used to model (with modifications) interest rate, and commodity price stochastically. An Ornstein-Uhlenbeck process $x(t)$, satisfies the following stochastic differential equation:

$$
d x(t)=\theta(\mu-x(t)) d t+\sigma d Z(t)
$$

where $\theta>0, \mu$ and $\sigma>0$ are parameters and $d Z(t)$ denotes the Wiener process. It is also mention a Vasicek model.

\subsection{Constant Elasticity of Variance (CEV) model}

In mathematical finance, the constant elasticity of variance $(\mathrm{CEV})$ model is a stochastic volatility model which attempt to capture stochastic volatility and the leverage effect. Therefore, the model is widely used by practitioners for modelling equities and commodities in financial industry. It was developed by John Cox in 1975. The constant elasticity of variance (CEV) model describes a process which evolves according to the following stochastic differential equation:

$$
d S(t)=\mu S(t) d t+\sigma S(t)^{\gamma} d Z(t)
$$

In which $S(t)$ is the spot price, $\mathrm{t}$ is time and $\mu$ is a parameter characterizing the drift, $\sigma$ and $\gamma$ are other parameters and $Z(t)$ is a Brownian motion. The notation " $d S(t)$ " represents a differential that is infinitesimally small change in parameter $S$.

The constant parameters $\sigma, \gamma$ satisfies the conditions $\sigma \geq 0, \gamma \geq 0$.

The parameter $\gamma$ controls the relationship between volatility and price, and is the central future of the model. When $\gamma<1$ we see the so-called leverage effect, commonly observed in equity markets where the volatility of a stock increases as its time falls. Conversely, in commodity markets, we often observed $\gamma>1$, the so-called inverse leverage effect where by the volatility of the price of a commodity tends to increase as its price increases.

\subsection{Dynamic Programming}

Dynamic programming or recursive optimization is a technique that is used for obtaining solutions for multistage decisions problems. There is no standard mathematical formulation of the dynamic programming for each problem depending on the variable given, and objective of the problem, one has to develop a particular equation to fit for solution. Nowadays, applications of dynamic programming are done in almost day to-day managerial problems, such as inventory problems, waiting line problems resource allocation and so on. Dynamic programming may be classified depending on the nature of data available as deterministic and stochastic or probabilistic models. In deterministic models, the outcome at any decision stage is uniquely determined and known. This technique was developed by Richard Bellman in the early (1950) principle of optimality: this principle implies that a wrong decision taken at a stage does not prevent from taking optimal decision for the remaining stages. That principle is the firm base for dynamic programming technique.

\subsection{Maximum Principle}

Maximum principle is used in optimal control theory to find the base possible control for taking a dynamical system from one state to another, especially in the presence of constraints for the state or input controls. It was formulated in (1956) by the Russian mathematician Lev Pontryagin and his students. It has as a special case the Euler-Lagrange equation of the calculus variations. The principle states, informally, that the control Hamiltonian must take an extreme value over control in the set of all admissible controls. Whether the extreme value is maximum or minimum depends both on the problem and on the sign convention used to defining the Hamiltonian. The normal convention, which is the one used in Hamiltonian leads to a maximum hence maximum principle but the sign convention used in this article makes the extreme value a minimum.

If $u$ is the set of values of permissible control, then the principle states that the optimal control $u^{*}$ must satisfy:

$$
\begin{gathered}
H\left(x^{*}(t), u^{*}(t), \lambda^{*}(t), t\right) \leq H\left(x^{*}(t), u, \lambda^{*}(t), t\right), \forall u \in U, t \\
\in\left[t_{o}, t_{f}\right]
\end{gathered}
$$

where

$x^{*} \in c^{1}\left[t_{o}, t_{f}\right]$ is the optimal state trajectory (a special type of optimization problem where the decision variables are functions rather than real numbers) and

$$
\lambda^{*} \in B \vee\left[t_{o}, t_{f}\right] \text { is the optimal costate trajectory. }
$$

\subsection{Hamilton-Jacobi-Bellman (HJB) Equation}

It is a partial differential equation which is central to optimal control theory. The solution of the HJB equation is the value function which gives the minimum cost for a given dynamical system with an associated cost function.

When solved locally, the Hamilton-Jacobi-Bellman is a 
necessary condition, but when solved over the whole of state space, the HJB equation is a necessary and sufficient condition for an optimum. The solution is open loop, but it also permits the solution of the closed loop problem. The Hamilton-Jacobi-Bellman equation can be generalized to stochastic system as well. The equation is a result of the theory of dynamic programming which was pioneered in the year (1950's) by Richard Bellman and co-workers.

\subsection{The Model Formation and the Model}

Assuming an investor trades two assets in a financial market: a risky asset (stock) and a risk free asset (bond) that has a rate that is a function of time. The dynamics of the price of the risk free asset denoted by $B(t)$ is given by;

$$
d B(t)=r(t) B(t) d t
$$

and

$$
\frac{d B(t)}{B(t)}=r(t) d t
$$

The risky asset is governed by the constant elasticity of variance $(\mathrm{CEV})$ model, stated as follows;

$$
d S(t)=S(t)\left[\mu d t+b S^{\gamma}(t) d Z_{1}(t)\right]
$$

from which we have

$$
\frac{d S(t)}{S(t)}=\left[\mu d t+b S^{\gamma}(t) d Z_{1}(t)\right]
$$

where $S(t)$ denotes the risky asset price at time $t$, $\mu, b$ andareconstants. $\mu$ is the appreciation rate of the risky asset $\{Z(t): t>0\}$ is a standard Brownian motion in a complete probability space $\left(\Omega, F,(F)_{t>0}, P\right) .(F)_{t>0}$ is the augmented filtration generated by the Brownian motion $Z(t)$. $b S^{\gamma}(t)$ is the instantaneous volatility and the elasticity $\gamma$, a parameter which satisfies the general condition $\gamma \leq 0$. If the elastic parameter $\gamma=0$, then equation (4) the constant elasticity of variance $\mathrm{CEV}$ model reduces to a geometric Brownian motion.

The Ornstein-Uhlebeck process is one of several approaches used to model (with modifications) interest rates, currency, exchange rates and commodity prices stochastically, it is given as;

$$
d r(t)=\theta(\mu-r(t)) d t+\sigma d Z_{2}(t) .
$$

Let $K(t)$ be the amount of money the investor puts in the risky asset at time $t$, then $[V(t)-K(t)]$ is the money amount he invested in the risk free asset, where $V(t)$ is the total money investment in both assets.

Corresponding to the trading strategy $K(t)$, the dynamics stochastic differential equation (SDE)

$$
d V(t)=K \frac{d S(t)}{S(t)}+[V(t)-K(t)] \frac{d B(t)}{B(t)}
$$

The substitution of (2) and (4) into equation (6) gives

$$
d V(t)=K\left[\mu d t+b S^{\gamma}(t) d Z_{1}(t)\right]+
$$

$$
[V(t)-K(t)] r(t) d t
$$

which simplifies to

$$
\begin{gathered}
d V(t)=\{(\mu-r(t)) K(t)+r(t) V(t)\} d t+ \\
b S^{\gamma}(t) K(t) d Z_{1}(t) .
\end{gathered}
$$

Let transaction cost be charged at the rate $c(t)$ on transactions involving only the risky asset, the equation (7) modifies to

$$
\begin{gathered}
d V(t)=K\left[\mu d t+b S^{\gamma}(t) d Z_{1}(t)\right]+(1-c(t)) \\
{[V(t)-K(t)] r(t) d t,}
\end{gathered}
$$

and equation (8) becomes

$$
\begin{gathered}
d V(t)=\left\{\begin{array}{c}
(\mu+(c(t)-1) r(t)) K(t)+ \\
(1-c(t)) r(t) V(t)
\end{array}\right\} d t \\
+b S^{\gamma}(t) K(t) d Z_{1}(t) .
\end{gathered}
$$

The quadratic variations of equations (8) and (10) is,

$$
[d V(t)]^{2}=b^{2} S^{2 \gamma}(t) K(t) d t
$$

Where

$$
\left.\begin{array}{c}
d t \cdot d t=d t \cdot d Z_{1}(t)=0 \\
d Z_{1}(t) \cdot d Z_{1}(t)=d t
\end{array}\right\} .
$$

The investor's problem is to find the optimal strategy for

$$
G(V, t ; T)=\operatorname{Max}_{K(t)} E[U(V)],
$$

subject to (8) when there is no transaction cost and (10) when there is transaction cost.

\section{The Optimization}

We assume the investor has exponential utility preference, that is,

$$
U(V)=a-\frac{b}{f} e^{-f v}
$$

with absolute risk aversion,

$$
-\frac{U^{\prime \prime}(V)}{U^{\prime}(V)}=f,
$$

where $V$ is the investor's wealth.

\subsection{A: When the Brownian Motions Do Not Correlate (That Is $\left.E\left(d z_{1} \cdot d z_{2}=o\right)\right)$ and There Is Transaction Cost}

We get the Bellman equation using the fact that,

$$
G(V, t ; T)=\operatorname{Max}_{K(t)} E\left[G\left(V^{\prime}, t ; T\right)\right]
$$

where $V^{\prime}$ is the wealth process at time $t+\Delta t$.

We get from equation (14) that 


$$
\operatorname{Max}_{K(t)} E\left[G\left(V^{\prime}, t ; T\right)-G(V, t ; T)\right]=0 .
$$

The division of equation (15) by $\Delta t$ and taking limit as $\Delta t$ tends to zero gives the Bellman equation as

$$
\operatorname{Max}_{K(t)} E\left[\frac{d G}{d t}\right]=0
$$

The maximum principle states that;

$$
d G=\frac{\partial G}{\partial t} d t+\frac{\partial G}{\partial s} d S+\frac{\partial G}{\partial r} d r+\frac{\partial G}{\partial v} d V+\frac{\partial^{2} G}{\partial s \partial r}(d S d r)+\frac{\partial^{2} G}{\partial s \partial v}(d S d V)+\frac{\partial^{2} G}{\partial r \partial v}(d r d V)+\frac{1}{2}\left[\frac{\partial^{2} G}{\partial s^{2}}(d S)^{2}+\frac{\partial^{2} G}{\partial r^{2}}(d r)^{2}+\frac{\partial^{2} G}{\partial v^{2}}(d V)^{2}\right]
$$

Now

$$
\left.\begin{array}{c}
d V(t)=\{(\mu+(c(t)-1) r(t)) K(t)+(1-c(t)) r(t) V(t)\} d t \\
+b S^{\gamma}(t) K(t) d Z_{1}(t), \\
d r(t)=\theta(\mu-r(t)) d t+\sigma d Z_{2}(t), \\
d S(t)=S(t)\left[\mu d t+b S^{\gamma}(t) d Z_{1}\right], \\
(d S(t))^{2}=b^{2} S^{2(\gamma+1)} d t \\
(d r(t))^{2}=\sigma^{2} d t, \\
(d V(t))^{2}=b^{2} S^{2 \gamma}(t) k^{2}(t) d t, \\
(d S(t) d V(t))=b^{2} S^{2 \gamma+1}(t) k(t) d t, \\
(d r d V)=0 \\
(d S d r)=0,
\end{array}\right\},
$$

where

$$
\left.\begin{array}{c}
d t \cdot d t=d t \cdot d Z_{1}=d t \cdot d Z_{2}=0 \\
d Z_{1} \cdot d Z_{1}=d Z_{2} \cdot d Z_{2}=d t \\
d Z_{1} \cdot d Z_{2}=0
\end{array}\right\} .
$$

Substituting for

$d S(t), d r(t), d V(t),(d S(t))^{2},(d r(t))^{2}$, and $(d V(t))^{2}$ into (19), we obtain

$$
\begin{array}{r}
d G=\frac{\partial G}{\partial t} d t+\frac{\partial G}{\partial s}\left\{S(t)\left[\mu d t+b S^{\gamma}(t) d Z_{1}\right]\right\}+\frac{\partial G}{\partial r}\left\{\alpha(\beta-r(t)) d t+\sigma d Z_{2}\right\}+\frac{\partial G}{\partial V}[\{(\mu+(c(t)-1) r(t)) K(t)+(1-c(t)) r(t) V(t)\} d+ \\
\left.b S^{\gamma}(t) K(t) d Z_{1}(t)\right]+\frac{1}{2}\left[\frac{\partial^{2} G}{\partial s^{2}}\left\{b^{2} S^{2(\gamma+1)}(t) d t\right\}+\frac{\partial^{2} G}{\partial r^{2}}\left\{\sigma^{2} d t\right\}+\frac{\partial^{2} G}{\partial V^{2}}\left\{b^{2} S^{2 \gamma}(t) k^{2}(t) d t\right\}\right]+\frac{\partial^{2} G}{\partial s \partial v}\left\{b^{2} S^{2 \gamma+}(t) k(t) d t\right\} .(22)
\end{array}
$$

Substituting equation (22) in equation (19) and taking the expectation, we get

$$
\begin{aligned}
G_{t}+G_{s} \mu S+ & G_{r}[\alpha(\beta-r)]+G_{s v}\left[b^{2} S^{(2 \gamma+1)} k\right] \frac{G_{r r}\left[\sigma^{2}\right]}{2}++\frac{G_{s s}\left[b^{2} S^{2(\gamma+1)}\right]}{2}+\frac{G_{v v}\left[b^{2} S^{2 \gamma} k^{2}\right]}{2} \\
& +G_{v}[\{(\mu+(c(t)-1) r(t)) K(t)+(1-c(t)) r(t) V(t)\}]=0,
\end{aligned}
$$

where

$$
E\left[d z_{1}\right]=E\left[d z_{2}\right]=0 .
$$

Differentiating (22) with respect to $k(t)$ gives

$$
G_{v}(\mu+(c(t)-1) r(t))+G_{s v}\left(b^{2} S^{(2 \gamma+1)}\right)+G_{v v}\left(b^{2} S^{2 \gamma} k(t)\right)=0,
$$

from which solving for $k(t)$ in equation (25), gives the optimal strategy

$$
k^{*}(t)=-\left[\frac{G_{v}(\mu+(c(t)-1) r(t))}{\left(b^{2} S^{2 \gamma}\right) G_{v v}}+\frac{S G_{s v}}{G_{v v}}\right] .
$$

Let

$$
G(t, r, s, v)=h(t, s, r)\left[a-\frac{b}{f} e^{-f v}\right]
$$

be a solution to equation (23), such that at the terminal time $T$

$$
h(T, s, r)=1,
$$

then we have from (27) that

$$
\left.\begin{array}{c}
G_{t}=h_{t}\left[a-\frac{b}{f} e^{-f v}\right], G_{s}=h_{s}\left[a-\frac{b}{f} e^{-f v}\right] \\
G_{r}=h_{r}\left[a-\frac{b}{f} e^{-f v}\right], \\
G_{v}=h\left[b e^{-f v}\right], G_{S v}=h\left[b e^{-f v}\right], G_{r v}=h_{r}\left[b e^{-f v}\right], \\
G_{S S}=h_{S S}\left[a-\frac{b}{f} e^{-f v}\right], \\
G_{r r}=h_{r r}\left[a-\frac{b}{f} e^{-f v},\right] G_{v v}=h\left[-f b e^{-f v}\right]
\end{array}\right\} .
$$

Using (29) in (26) substituting for $G_{v}, G_{s v}$, and $G_{v v}$ and simplifying we obtain the investor's optimal strategy a

$$
k_{n c \& t}{ }^{*}(t)=\left[\frac{(\mu+(c(t)-1) r(t))+b^{2} S^{(2 \gamma+1)}}{f b^{2} S^{2 \gamma}}\right] .
$$


Clearly when there is no transaction cost (that is when $c(t)=0$ ) the optimal strategy becomes

$$
k_{n c \& n t}{ }^{*}(t)=\left[\frac{(\mu-r(t))+b^{2} S^{(2 \gamma+1)}}{f b^{2} S^{2 \gamma}}\right] .
$$

\subsection{B: When the Brownian Motions Correlate (That Is $\left.E\left[d z_{1} \cdot d z_{2}\right]=\rho d t\right)$ and There Is Transaction Cost}

In this case equation (18) becomes

$$
\left.\begin{array}{c}
d V(t)=\{(\mu+(c(t)-1) r(t)) K(t)+(1-c(t)) r(t) V(t)\} d t \\
+b S^{\gamma}(t) K(t) d Z_{1}(t), \\
d r(t)=\theta(\mu-r(t)) d t+\sigma d Z_{2}(t), \\
d S(t)=S(t)\left[\mu d t+b S^{\gamma}(t) d Z_{1}\right], \\
(d S(t))^{2}=b^{2} S^{2(\gamma+1)} d t, \\
(d r(t))^{2}=\sigma^{2} d t \\
(d V(t))^{2}=b^{2} S^{2 \gamma}(t) k^{2}(t) d t, \\
(d S(t) d V(t))=b^{2} S^{2 \gamma+1}(t) k(t) d t, \\
(d r(t) d V(t))=\rho \sigma b S^{\gamma}(t) k(t) d t, \\
(d S(t) d r(t))=\rho \sigma b S^{(\gamma+1)}(t) d t,
\end{array}\right\}
$$

Using equation (32) into equation (22), we get

$$
\begin{gathered}
d G=\frac{\partial G}{\partial t} d t+\frac{\partial G}{\partial s}\left\{S(t)\left[\mu d t+b S^{\gamma}(t) d Z_{1}\right]\right\}+\frac{\partial G}{\partial r}+\frac{\partial^{2} G}{\partial s \partial v}\left\{b^{2} S^{2 \gamma+}(t) k(t) d t\right\}+\frac{\partial^{2} G}{\partial s \partial r}\left\{\rho \sigma b S^{(\gamma+1)}(t) d t\right\} \\
+\frac{\partial^{2} G}{\partial r \partial v}\left\{\rho \sigma b S^{\gamma}(t) k(t) d t\right\}+\frac{\partial G}{\partial V}\left[\{(\mu+(c(t)-1) r(t)) K(t)+(1-c(t)) r(t) V(t)\} d t+b S^{\gamma}(t) K(t) d Z_{1}(t)\right] \\
+\frac{1}{2}\left[\frac{\partial^{2} G}{\partial s^{2}}\left\{b^{2} S^{2(\gamma+1)}(t) d t\right\}+\frac{\partial^{2} G}{\partial r^{2}}\left\{\sigma^{2} d t\right\}+\frac{\partial^{2} G}{\partial V^{2}}\left\{b^{2} S^{2 \gamma}(t) k^{2}(t) d t\right\}\right]
\end{gathered}
$$

with which we obtain the new Hamilton-Jacobi-Bellman (HJB) equation from (23) to be

$$
\begin{aligned}
G_{t}+G_{s} \mu S+ & G_{v}[\{(\mu+(c(t)-1) r(t)) K(t)+(1-c(t)) r(t) V(t)\}]+G_{r}[\alpha(\beta-r)]+G_{s v}\left[b^{2} S^{(2 \gamma+1)} k\right] \\
& +\frac{G_{s S}\left[b^{2} S^{2(\gamma+1)}\right]}{2}+\frac{G_{r r}\left[\sigma^{2}\right]}{2}+\frac{G_{v v}\left[b^{2} s^{2 \gamma} k^{2}\right]}{2}+G_{r v}\left[\rho \sigma b S^{\gamma} k\right]+G_{r v}\left[\rho \sigma b S^{(\gamma+1)}\right]=0,
\end{aligned}
$$

where

$$
E\left[d z_{1}\right]=E\left[d z_{2}\right]=0 .
$$

Making use of the first principle, differentiating (34) with respect to $k(t)$ gives

$$
G_{v}(\mu+(c(t)-1) r(t))+G_{s v}\left(b^{2} S^{(2 \gamma+1)}\right)+G_{r v}\left(\rho \sigma b S^{\gamma}\right)+G_{v v}\left(b^{2} S^{2 \gamma} k\right)=0 .
$$

Solving for $k(t)$ in equation (36), we ge

$$
k^{*}(t)=-\left[\frac{G_{v}(\mu+(c(t)-1) r(t))+G_{s v}\left(b^{2} S^{(2 \gamma+1)}\right)+(\rho \sigma) G_{r v}}{\left(b^{2} S^{2 \gamma}\right) G_{v v}}\right] .
$$

Now, using equation (27)-(29) in equation (37) and simplifying, we obtain

$$
k^{*}(t)=-\left[\frac{(\mu+(c(t)-1) r(t))}{f b^{2} S^{2 \gamma}}+\frac{S}{f}+\frac{\rho \sigma h_{r}}{f b^{2} S^{2 \gamma}}\right] .
$$

To eliminate the dependency on $r$ in (38), we let

$$
h(t, s, r)=w(t, s)\left[-\frac{b}{f} e^{-f r}\right]
$$

such that at the terminal time $T$

$$
w(T, s)=\frac{-f e^{f r}}{b} .
$$

From (39), we obtain

$$
h_{r}=w(t, s)\left[-b e^{-f r}\right],
$$

which is applied in (38) and with simplification we obtain the investor's optimal strategy as

$$
k_{c \& t}{ }^{*}(t)=\left[\frac{(\mu+(c(t)-1) r(t))+b^{2} S^{(2 \gamma+1)}+f \rho \sigma}{f b^{2} S^{2 \gamma}}\right] .
$$

Here also, when there is no transaction cost the optimal investment strategy is

$$
k_{c \& n t}^{*}(t)=\left[\frac{(\mu-r(t))+b^{2} s^{(2 \gamma+1)}+f \rho \sigma}{f b^{2} s^{2 \gamma}}\right] .
$$

\subsection{The Effects Transaction Cost and the Correlation of}

\section{Brownian motions}

To investigate the effects transaction cost and the correlation of Brownian motions, based on the following four results obtained: (a) When the Brownian motions do not correlate and there is transaction cost, (b) When the Brownian motions do not correlate and there is no transaction cost, (c) When the Brownian motions correlate and there is transaction cost, and (d) When the Brownian motions correlate and there is no transaction cost, we consider the cases:

(1) When the Brownian motions do not correlate and there is transaction cost and when the Brownian motions do not correlate and there is no transaction cost.

(2) When the Brownian motions do not correlate and there is transaction cost and when the Brownian motions correlate 
and there is transaction cost.

(3) When the Brownian motions do not correlate and there is transaction cost and when the Brownian motions correlate and there is no transaction cost.

(4) When the Brownian motions do not correlate and there is no transaction cost and when the Brownian motions correlate and there is transaction cost.

(5) When the Brownian motions do not correlate and there is no transaction cost and when the Brownian motions correlate and there is no transaction cost.

(6) When the Brownian motions correlate and there is transaction cost and when the Brownian motions correlate and there is no transaction cost.

\subsubsection{CASE 1: When the Brownian Motions do Not Correlate and There Is Transaction Cost and When the Brownian Motions do Not Correlate and There Is No Transaction Cost}

The optimal investment strategy when the Brownian motions do not correlate a and there is transaction cost is given by equation (30)

$$
k_{n c \& t}^{*}(t)=\left[\frac{(\mu+(c(t)-1) r(t))+b^{2} S^{(2 \gamma+1)}}{f b^{2} S^{2 \gamma}}\right] .
$$

and by equation (31)

$$
k_{n c \& n t}^{*}(t)=\left[\frac{(\mu-r(t))+b^{2} s^{(2 \gamma+1)}}{f b^{2} s^{2 \gamma}}\right] .
$$

when the Brownian motions do not correlate and there is no transaction cost.

From the above we have

$$
k_{n c \& t}^{*}(t)=\left\{\frac{(\mu-r(t))+c(t) r(t)+b^{2} S^{(2 \gamma+1)}}{f b^{2} S^{2 \gamma}}\right\}
$$

from which

$$
k_{n c \& t}^{*}(t)=k_{n c \& n t}^{*}(t)+\frac{c(t) r(t)}{f b^{2} S^{2 \gamma}} .
$$

This shows that the investor has an optimal investment strategy that requires more amount of money valuing $\frac{c(t) r(t)}{f b^{2} S^{2 \gamma}}$ for investment when the Brownian motions do not correlate and there is transaction cost than when the Brownian motions do not correlate and there is no transaction.

\subsubsection{CASE 2: When the Brownian Motions do Not Correlate and There Is Transaction Cost and When the Brownian Motions Correlate and There Is Transaction Cost}

For this case we have from equation (30)

$$
k_{n c \& t}^{*}(t)=\left[\frac{(\mu+(c(t)-1) r(t))+b^{2} S^{(2 \gamma+1)}}{f b^{2} S^{2 \gamma}}\right]
$$

and from equation (42)

$$
k_{c \& t}{ }^{*}(t)=\left[\frac{(\mu+(c(t)-1) r(t))+b^{2} S^{(2 \gamma+1)}+f \rho \sigma}{f b^{2} S^{2 \gamma}}\right] .
$$

It can be seen that

$$
k_{c \& t}{ }^{*}(t)=k_{n c \& t}^{*}(t)+\frac{\rho \sigma}{b^{2} s^{2 \gamma}} .
$$

So the investor has an optimal investment strategy that requires more amount of money valuing $\frac{\rho \sigma}{b^{2} S^{2 \gamma}}$ for investment when the Brownian motions do correlate and there is transaction cost than when the Brownian motions do not correlate and there is transaction.

\subsubsection{CASE 3: When the Brownian Motions do Not Correlate and There Is Transaction Cost and When the Brownian Motions Correlate and There Is no Transaction Cost}

Here we have equation (30)

$$
k_{n c \& t}{ }^{*}(t)=\left[\frac{(\mu+(c(t)-1) r(t))+b^{2} S^{(2 \gamma+1)}}{f b^{2} S^{2 \gamma}}\right]
$$

and equation (43)

$$
k_{c \& n t}{ }^{*}(t)=\left[\frac{(\mu-r(t))+b^{2} S^{(2 \gamma+1)}+f \rho \sigma}{f b^{2} S^{2 \gamma}}\right] .
$$

We now have

$$
\begin{aligned}
k_{n c \& t}{ }^{*}(t) & =\left[\frac{(\mu+(c(t)-1) r(t))+b^{2} S^{(2 \gamma+1)}}{f b^{2} S^{2 \gamma}}\right] \\
& =k_{c \& n t}{ }^{*}(t)+\frac{[c(t) r(t)-f \rho \sigma]}{f b^{2} S^{2 \gamma}} .
\end{aligned}
$$

This implies that the investor has an optimal investment strategy that requires more amount of money valuing $\frac{[c(t) r(t)-f \rho \sigma]}{f b^{2} s^{2 \gamma}}$ for investment when the Brownian motions do not correlate and there is transaction cost than when the Brownian motions correlate and there is no transaction.

\subsubsection{CASE 4: When the Brownian Motions Do Not} Correlate and There Is no Transaction Cost and When the Brownian Motions Correlate and There Is Transaction Cost

Equation (31) gives

$$
k_{n c \& n t}{ }^{*}(t)=\left[\frac{(\mu-r(t))+b^{2} S^{(2 \gamma+1)}}{f b^{2} s^{2 \gamma}}\right],
$$

for when the Brownian motions do not correlate and there is no transaction cost. Equation (42) gives, for when the Brownian motions correlate and there is transaction cost,

$$
k_{c \& t}{ }^{*}(t)=\left[\frac{(\mu+(c(t)-1) r(t))+b^{2} S^{(2 \gamma+1)}+f \rho \sigma}{f b^{2} S^{2 \gamma}}\right] .
$$

From above we have 


$$
\begin{aligned}
k_{c \& t}{ }^{*}(t) & =\left[\frac{(\mu+(c(t)-1) r(t))+b^{2} S^{(2 \gamma+1)}+f \rho \sigma}{f b^{2} S^{2 \gamma}}\right] \\
& =k_{n c \& n t}{ }^{*}(t)+\frac{c(t) r(t)+f \rho \sigma}{f b^{2} S^{2 \gamma}} .
\end{aligned}
$$

Clearly equation (47) shows that the investor requires more money to be in business when the Brownian motions correlate and there is transaction cost than when the Brownian motions do not correlate and there is no transaction cost with the value $\frac{c(t) r(t)+f \rho \sigma}{f b^{2} S^{2 \gamma}}$

\subsubsection{CASE 5: When the Brownian Motions Do Not \\ Correlate and There Is no Transaction}

cost and when the Brownian motions correlate and there is no transaction

cost.

This case will be investigated using equation (31), when the Brownian motions do not correlate and there is no transaction cost

$$
k_{n c \& n t} *(t)=\left[\frac{(\mu-r(t))+b^{2} s^{(2 \gamma+1)}}{f b^{2} s^{2 \gamma}}\right],
$$

and equation (43)

$$
k_{c \& n t}{ }^{*}(t)=\left[\frac{(\mu-r(t))+b^{2} S^{(2 \gamma+1)}+f \rho \sigma}{f b^{2} S^{2 \gamma}}\right] .
$$

for when the Brownian motions correlate and there is no transaction cost.

We now have

$$
\begin{aligned}
k_{c \& n t}{ }^{*}(t) & =\left[\frac{(\mu-r(t))+b^{2} S^{(2 \gamma+1)}+f \rho \sigma}{f b^{2} S^{2 \gamma}}\right] \\
& =k_{n c \& n t}{ }^{*}(t)+\frac{\rho \sigma}{b^{2} S^{2 \gamma}} .
\end{aligned}
$$

Here we find that the investor needs more money for investment when the Brownian motions correlate and there is no transaction cost than when the Brownian motions do not correlate and there is no transaction cost. This difference is $\frac{\rho \sigma}{b^{2} S^{2 \gamma}}$.

\subsubsection{CASE 6: When the Brownian Motions Correlate and There Is Transaction Cost and When the Brownian Motions Correlate and There Is No Transaction Cost}

We have from equation (42) when the Brownian motions correlate and there is transaction cost that

$$
k_{c \& t}^{*}(t)=\left[\frac{(\mu+(c(t)-1) r(t))+b^{2} S^{(2 \gamma+1)}+f \rho \sigma}{f b^{2} S^{2 \gamma}}\right],
$$

and from equation (43) when the Brownian motions correlate there is no transaction cost that the optimal investment strategy is

$$
k_{c \& n t}{ }^{*}(t)=\left[\frac{(\mu-r(t))+b^{2} S^{(2 \gamma+1)}+f \rho \sigma}{f b^{2} S^{2 \gamma}}\right] .
$$

Now considering these equations we get

$$
\begin{aligned}
k_{c \& t}{ }^{*}(t) & =\left[\frac{(\mu+(c(t)-1) r(t))+b^{2} S^{(2 \gamma+1)}+f \rho \sigma}{f b^{2} S^{2 \gamma}}\right] \\
& =k_{c \& n t}{ }^{*}(t)+\frac{c(t) r(t)}{f b^{2} S^{2 \gamma}} .
\end{aligned}
$$

Equation (49) shows that the money the investor requires for investment when the Brownian motions correlate and there is transaction cost is more than the money he requires when the Brownian motions correlate and there is no transaction cost by $\frac{c(t) r(t)}{f b^{2} S^{2 \gamma}}$.

It can be observed that cases (1) and (6) have a pattern that resemble. Also cases (2) and (5).

Cases (3) and (4) are reverse of each other.

\section{Conclusion}

This study considered the investment strategy problem of an investor who has exponential utility preference and traded two assets (risky and risk-free assets). The price mechanism of the risky asset is governed by the constant elasticity of variance model while that of the risk-free asset follow the Ornstein-Uhlenbeck model.

The optimal strategies for the cases investigated are obtained starting with the Bellman equation from which the second order partial differential equations, the Hamilton-Jacobi-Bellman (H-J-B) equations, are derived. The methods of first principle and elimination of dependency on variables are employed to the get strategies that are optimal for the cases considered where the Brownian motions correlate and where they do not correlate. Also, the cases of charging and not charging transaction cost are looked into.

Among the results obtained in respect of the impacts of the correlation and none correlation of Brownian motions and paying and not paying transaction cost is that the investor needs more money for investment when the Brownian motions correlate and there is no transaction cost than when the Brownian motions do not correlate and there is no transaction cost with the difference $\frac{\rho \sigma}{b^{2} S^{2 \gamma}}$.

\section{References}

[1] Merton R. C. (1971). Optimum Consumption and Portfolio Rules in a Continuous-time model, Journal of Economic Theory, Vol. 3, No. 4, 373-413.

[2] Gu A., Guo X., and Li Z. (2012). Optimal Control of excess-of-loss Reinsurance an Investment for Insurers under Constant Elasticity of variance (CEV) model, Insurance Mathematics and Economics, Vol. 51, No. 3, 674-684.

[3] Liu J., Bai L., and Yiu K. C. (2012). Optimal Investment with a value-at-risk constraint. Journal of Industrial and Management Optimization [JIMO], 8 (3): 531-457

[4] Zhao H., Rong X. (2012): Portfolio selection problem with multiple risky assets under the constant elasticity of variance model. Elsevier, vol. 50 (1), pages 179-190. 
[5] Chang H., Chang K. and Lu J. M. (2014): Portfolio selection with liability and affine interest rate in the HARA utility framework, Abstract and Applied Analysis. 2014, pp. 1-12

[6] Ihedioha S. A, Oruh B. I. \& Osu B. O. (2017). Effects of Correlation of Brownian Motions on an Investor's Optimal Investment and Consumption Decision under Ornstein-Uhlenbeck Model, Academic Journal of Applied Mathematical Sciences, Academic Research Publishing Group, Vol 3 (6), pages 52-61, 06-2017. Handle: RePEc: arp: ajoams: 2017: 52-61.

[7] Okonkwo, C. U., Osu B. O., Ihedioha S. A. and Chibuisi C. (2018). Optimal Investment Strategy for Defined Contribution Pension Scheme under the Heston Volatility Model, Journal of Mathematical Finance, 8, 613-622

[8] Osu B. O., Njoku K. N. C and Basimanebotlhe O. S. (2019). Fund Management Strategies for a Defined Contribution (DC) Pension Scheme under the Default Fund Phase IV, Communications in Mathematical Finance, vol. 8, no. 1, 2019, 169-185 ISSN: 2241-195X (print), 2241- 1968 (online) Scientific Press International Limited.

[9] Akpanibah E. E., Osu B. O., Njoku K. N. C and Eyo O. A (2017). Optimization of Wealth Investment Strategies for DCPension Fund with Stochastic Salary and Extra Contributions, International Journal of Partial Differential Equations and Applications, vol. 5. no. 1, 33-41.

[10] Njoku K. N. C., Osu B. O., Akpanibah E. E. and Ujumadu R. N (2017). Effect of Extra Contribution on Stochastic Optimal Investment Strategies for DC Pension with Stochastic Salary under the Affine Interest Rate Model, Journal of Mathematical Finance, 7, 821-833.
[11] Wang L. and Chen, Z. (2018). Nash Equilibrium Strategy for a DC Pension Plan with State Dependent Risk Aversion: A Multiperiod Mean-Variance Framework. Hindawi Discrete Dynamics in Nature and Society Article ID 7581231 https://doi.org/10.1155/2018/7581231.

[12] Osu B. O., Akpanibah E. E. and Olunkwa C. (2018). Mean-Variance Optimization of portfolios with return of premium clauses in a DC pension plan with multiple contributors under constant elasticity of variance model, Int. J. Math. Comput. Sci., 12 (2018), 85-90. 1

[13] Osu B. O., Akpanibah E. E. and Oruh B. I. (2017). Optimal investment strategies for Defined Contribution (DC) pension fund with multiple contributors via Legendre transform and dual theory, Int. J. Pure Appl. Res., 2 97-105. 1

[14] Osu B. O., Njoku K. N. C. and Oruh B. I. (2019). On the Effect of Inflation and Impact of Hedging on Pension Wealth Generation Strategies under the Geometric Brownian motion Mode, Earthline Journal of Mathematical Sciences, Volume 1, Number 2, 2019, Pages 119-142. http://www.earthlinepublishers.com; https://doi.org/10.34198/ejms.1219.119142

[15] Njoku K. N. C. and Osu B. O. (2019). On the Modified Optimal Investment Strategy for Annuity Contracts under the Constant Elasticity of Variance (CEV) Model, Earthline Journal of Mathematical Sciences, Volume 1, Number 1, 63-90. $\mathrm{http}: / / \mathrm{www}$.earthlinepublishers.com, https://doi.org/10.34198/ejms.1119.6390 